\title{
Exploring the Effects of Technology-Enabled Mindfulness and Meditation on Stress Management
}

Nils Fischer ${ }^{1}$; Stephen Agboola ${ }^{1,2}$; Jennifer Felsted ${ }^{1,2}$; Sujay Kakarmath ${ }^{1,2}$; Sara Bersche Golas ${ }^{1}$; Joseph Kvedar ${ }^{1,2}$; Kamal Jethwani ${ }^{1,2}$

${ }^{1}$ Partners Connected Health, Partners HealthCare, Boston, MA, United States

${ }^{2}$ Harvard Medical School, Boston, MA, United States

Corresponding Author:

Nils Fischer

Partners Connected Health

Partners HealthCare

25 New Chardon Street

Boston, MA,

United States

Phone: 16439958

Email: nfischer@partners.org

\section{Abstract}

Background: Studies suggest that certain technologies may help regulate emotional states and reduce stress. Spire is a wearable device that measures breathing patterns to identify distinct emotional states—calm, tense or focus—to send feedback to the user. Muse is a meditation device that gives real time user audio-feedback based on the EEG wave patterns.

Objective: The objective of our study was to test the effect of each device on managing stress.

Methods: There were 126 participants recruited remotely and randomized to one of two interventions evaluating the Spire (group 1) and Muse (group 2) devices. Each study involved a 2-week baseline period followed by a 6-week intervention. All participants wore the Spire device in the baseline period where it collected data on the frequency and duration of calm, focus, or tense breathing patterns. Then, in group 1, feedback from the Spire device was turned on during the intervention period alerting participants of unfavorable breathing patterns on a companion app. In group 2, feedback from the Spire device remained off for the 6-week intervention period. Additionally, participants in group 2 meditated with the Muse device for 3 to 5 times per week during the intervention period. The Perceived Stress Survey (PSS-14) and Connor-Davidson Stress Resilience Scale (CD-Risc) were administered to all participants at enrollment (Week 1) and closeout (Week 8). Questionnaire and device data from group 1 and 2 were analyzed to determine the effect of each device on mental health outcomes from enrollment to closeout, and baseline to intervention, respectively.

Results: In group 1, perceived stress significantly decreased from 23.59 to $20.24(P=.001)$, but there was no significant change in stress resilience [(68.25 to $69.5(P=.44)]$. In group 2, perceived stress significantly decreased from 22.49 to $19.15(P<.001)$, and stress resilience scores significantly increased from 70.00 to $73.44(P=.014)$. Average calm minutes per day using Spire did not significantly change for Group 1 or 2 [61.75 (SD 27.25) to 59.22 (SD 35.24) (P=.69) and 69.72 (SD 29.41) to 63.89 (SD 29.71) $(P=.13)$, respectively]. In Group 1 , average focus minutes per day using Spire significantly decreased from 64.15 (SD 31.2) to 50.79 (SD 28.73) ( $P=.001)$ minutes, but in group 2, the average focus minutes per day as measured Spire did not significantly change [82.38 (SD) to 71.29 (SD 46.55) $(P=.08)]$. In Group 1, average tense streaks did not significantly decrease [17.63 (SD 16.99) to 14.08 (SD 10.63) ( $P=.15)$ ]. However, in Group 2, average tense streaks significantly decreased from 18.67 $(\mathrm{SD} 16.38)$ to 13.78 (SD 11.71) $(P=.004)$. After controlling for age, minutes of Spire use, baseline depression, and baseline anxiety in group 1 participants, each day of Spire use was significantly associated with $53.04(P=.02)$ more minutes of calm and 62.69 $(P=.04)$ more minutes of focus mind-states. Group 2 participants had 2.9 more tense mind-state minutes $(P=.02)$ with each day of using the Muse device after also controlling for minutes of Muse use, baseline depression, baseline anxiety and age.

Conclusions: Emotion sensing technologies may have a positive role in improving stress management.

(iproc 2017;3(1):e23) doi: 10.2196/iproc.8463 


\section{Multimedia Appendix 1}

Full paper.

[PDF File (Adobe PDF File), 820KB-Multimedia Appendix 1]

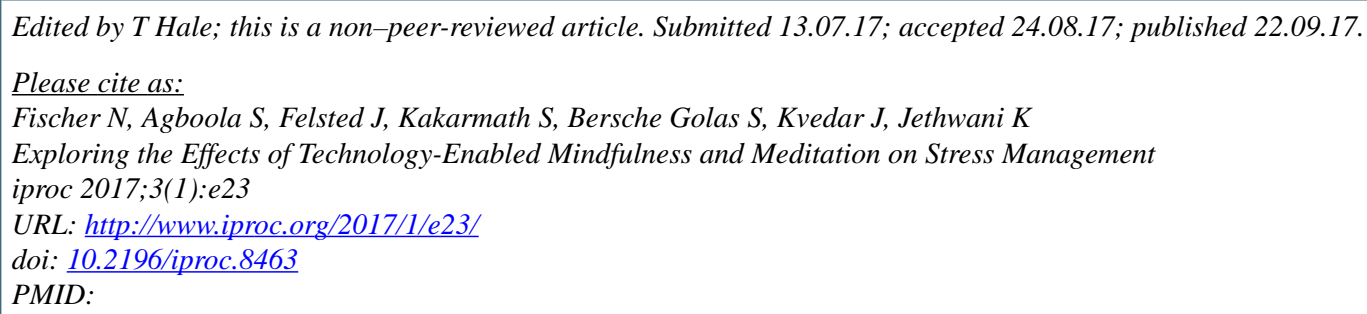

Edited by T Hale; this is a non-peer-reviewed article. Submitted 13.07.17; accepted 24.08.17; published 22.09.17.

Please cite as:

Fischer N, Agboola S, Felsted J, Kakarmath S, Bersche Golas S, Kvedar J, Jethwani K

Exploring the Effects of Technology-Enabled Mindfulness and Meditation on Stress Management iproc 2017;3(1):e23

URL: http://www.iproc.org/2017/1/e23/

doi: 10.2196/iproc.8463

PMID:

CNils Fischer, Stephen Agboola, Jennifer Felsted, Sujay Kakarmath, Sara Bersche Golas, Joseph Kvedar, Kamal Jethwani. Originally published in Iproceedings (http://www.iproc.org), 22.09.2017. This is an open-access article distributed under the terms of the Creative Commons Attribution License (https://creativecommons.org/licenses/by/4.0/), which permits unrestricted use, distribution, and reproduction in any medium, provided the original work, first published in Iproceedings, is properly cited. The complete bibliographic information, a link to the original publication on http://www.iproc.org/, as well as this copyright and license information must be included. 